\title{
Alterations in aerobic energy expenditure and neuromuscular function during a simulated cross-country skiathlon with the skating technique
}

\author{
Nicolas Fabre ${ }^{\mathrm{a}, *}$, Laurent Mourot ${ }^{\mathrm{b}, \mathrm{c}}$, Chiara Zoppirolli ${ }^{\mathrm{d}}$, Erik Andersson ${ }^{\mathrm{a}}$, \\ Sarah J. Willis ${ }^{\text {a }}$, Hans-Christer Holmberg ${ }^{\mathrm{a}}$ \\ ${ }^{a}$ Swedish Winter Sports Research Centre, Department of Health Sciences, Mid Sweden University, Östersund, Sweden \\ ${ }^{\mathrm{b}}$ Research Unit EA4660, Culture Sport Health Society and Exercise Performance Health Innovation Platform, Franche-Comté \\ University, Besançon, France \\ ${ }^{\mathrm{c}}$ Clinical Investigation Centre, INSERM CIT 808, CHRU of Besançon, France \\ ${ }^{\mathrm{d}}$ CeRiSM, Center of Research in Mountain Sport and Health, Department of Neurological, Neuropsychological, Morphological \\ and Movement Sciences, University of Verona, Rovereto, Italy
}

\section{A R T I C L E I N F O}

\section{Article history:}

Available online 11 February 2015

PsycINFO classification:

2300

2330

Keywords:

Cross-country skiing

EMG

Muscle fatigue

Oxygen uptake

Techniques

Transition

\begin{abstract}
A B S T R A C T
Here, we tested the hypothesis that aerobic energy expenditure (AEE) is higher during a simulated 6-km (2 loops of 3-km each) "skiathlon" than during skating only on a treadmill and attempted to link any such increase to biomechanical and neuromuscular responses. Six elite male cross-country skiers performed two pretesting time-trials (TT) to determine their best performances and to choose an appropriate submaximal speed for collection of physiological, biomechanical and neuromuscular data during two experimental sessions (exp). Each skier used, in randomized order, either the classical (CL) or skating technique (SK) for the first 3-km loop, followed by transition to the skating technique for the second 3-km loop. Respiratory parameters were recorded continuously. The EMG activity of the triceps brachii (TBr) and vastus lateralis (VLa) muscles during isometric contractions performed when the skiers were stationary (i.e., just before the first loop, during the transition, and after the second loop); their corresponding activity during dynamic contractions; and pole and plantar forces during the second loop were recorded. During the second 3-km
\end{abstract}

* Corresponding author at: Swedish Winter Sports Research Centre, Department of Health Sciences, Mid Sweden University, 83125 Östersund, Sweden. Tel.: +33 (0)6 08093194.

E-mail address: colsfabre@gmail.com (N. Fabre). 
of the TT, skating speed was significantly higher for the SK-SK than CL-SK. During this second loop, AEE was also higher $(+1.5 \%)$ for

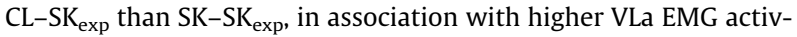
ity during both isometric and dynamic contractions, despite no differences in plantar or pole forces, poling times or cycle rates. Although the underlying mechanism remains unclear, during a skiathlon, the transition between the sections of classical skiing and skating alters skating performance (i.e., skiing speed), AEE and neuromuscular function.

(c) 2015 Elsevier B.V. All rights reserved.

\section{Introduction}

Recent developments in cross-country skiing (XCS) competitions include "skiathlon" events in which male and female skiers race a total distance of $30 \mathrm{~km}$ and $15 \mathrm{~km}$, respectively, using the classic XCS technique (CL) in the first half and the skating technique (SK) in the second. This requires a change in technique and is thereby in some ways comparable to triathlon events in which athletes goes through transitions from swimming to cycling and cycling to running.

In the triathlon, the well-documented physiological, biomechanical and neuromuscular responses following a transition from cycling to running (Millet \& Vleck, 2000) indicate that the energetic cost of running after a bout of cycling is as much as $12 \%$ greater than for running with or without a prior bout of running (Guezennec, Vallier, Bigard, \& Durey, 1996; Hausswirth, Bigard, Berthelot, Thomaidis, \& Guezennec, 1996; Hue, Le Gallais, Chollet, Boussana, \& Prefaut, 1998; Kreider, Boone, Thompson, Burkes, \& Cortes, 1988). It has been suggested that a prior bout of cycling alters running kinematics in a manner that enhances increased internal mechanical work, thus increasing the energetic cost (Hausswirth, Bigard, \& Guezennec, 1997).

Reductions in neuromuscular coordination and sensory inputs have also been proposed to be involved in this increased energy cost of running (Quigley \& Richards, 1996). Theoretically, such changes in muscle activation may be explained by the selection of a different, but not necessarily less efficient or less skilled pattern of movement, rather than less skilled execution of the same task. On the basis of limb kinematics alone, Quigley and Richards (1996) concluded that prior cycling does not influence control of movement during subsequent running. However, these investigators did not consider the redundancy of the motor control system and the possibility that pelvic and limb movements might persist even when muscle activity is altered. Although the reasons for such lack of coordination remain unclear, interference with the control of movement and muscle activity due to repeated prior performance of cycling is likely to contribute (Heiden \& Burnett, 2003). Various authors have also suggested that triathletes who perform better demonstrate less change in their energetic cost of running (Hue et al., 1998; Millet, Millet, Hofmann, \& Candau, 2000).

A general principle of human movement is to attempt to minimize metabolic energy expenditure. For example, when running at a given speed, each individual has an optimal stride frequency that minimizes this expenditure (Cavanagh \& Williams, 1982) and experienced runners automatically adopt this frequency. However, when a non-optimal stride frequency is imposed and then released, runners demonstrate a bi-phasic delay in returning to their optimal stride frequency (Snyder, Snaterse, \& Donelan, 2012). During this delay, the metabolic cost is presumably greater and performance thus impaired. Transitions between modes of locomotion (e.g., cycling to running) or between different techniques of XCS may involve a similar delay in re-optimization of metabolic cost and performance.

To the best of our knowledge, no comparable data for the transition from the CL to SK technique of skiing during a skiathlon are available. On the basis of the triathlon studies and because the CL and SK techniques involve different patterns of movement (Bilodeau, Boulay, \& Roy, 1992), we hypothesized that the aerobic energy expenditure (AEE) of skating would be higher following transition from the 
classical technique during a simulated skiathlon (CL-SK) than when there is no change in technique [skating alone (SK-SK)]. We also attempted to link any such difference to altered physiological, biomechanical and neuromuscular responses.

\section{Methods}

\subsection{Participants}

Six elite male cross-country skiers provided their written consent to participate in this study, which was pre-approved by the Regional Ethical Review Board in Umeå, Sweden. They all competed at the national level in Sweden and had previous experience of roller-skiing on a treadmill for performance testing and training. Their age was $27 \pm 6$ years; height $179.6 \pm 8.3 \mathrm{~cm}$; body mass $77.4 \pm 10.4 \mathrm{~kg}$; peak oxygen uptake $\left(\mathrm{V}_{2}\right.$ peak $)$ recorded during the present testing sessions $4.9 \pm 0.5 \mathrm{~L} \mathrm{~min}^{-1}$; and performance level (International Ski Federation [FIS] points from FIS distance races (a skier's rank is relative to a 0-point standard established by the top-ranked skier in the world) $100.7 \pm 22.5$ (mean \pm SD). The participants refrained from ingesting caffeine or alcohol for at least $12 \mathrm{~h}$ before testing, ate a light meal $3 \mathrm{~h}$ before testing, and refrained from strenuous exercise before all testing sessions.

\subsection{Experimental design}

All tests were performed within a 15-day period on a motor-driven treadmill specially designed for roller-skiing (belt dimensions $2.5 \mathrm{~m} \times 3.5 \mathrm{~m}$, Rodby, Södertalje, Sweden). Each participant used the same pair of roller skis, which were pre-warmed in a heating box before each test to avoid a warmup effect on the wheels and bearings during testing. The skiers used Pro-Ski (Sterners, Nyhammar, Sweden) roller-skis (model C2 and S2 for the classic and skating styles, with coefficients of rolling resistance of 0.0220 and 0.0227 , respectively). During all treadmill rolling-skiing sessions and tests, the participants wore a safety harness suspended from the ceiling.

All participants completed four testing sessions on four different days with at least a 24-h recovery period between sessions. All test sessions were performed at the same time of day to minimize diurnal variability. Two preliminary time-trials $(2 \times 3-\mathrm{km}$ each, CL-SK $\mathrm{TT}$ and SK-SK $\mathrm{TT})$ performed at freely chosen race speed, served to determine each individual's sub-maximal roller-skiing speed, which was defined as $96 \%$ of the speed on each segment of the track (see Section 2.2.2). These pre-determined speeds would later be used for data collection during the two experimental sessions (CL-SK $\mathrm{exp}_{\text {. }}$ and SK-SK exp ). During these sessions, the speed was the same and submaximal in order to obtain more stable and comparable measurements. An overview of the experimental procedure for collection of data is illustrated in Fig. 1.

\subsubsection{Preliminary testing (sessions 1 and 2)}

In the two first time-trials (TT), performed in random order, the participants were asked to rollerski as fast as possible over a simulated 6-km course divided into two loops of $3 \mathrm{~km}$ each. In one of these time-trials (CL-SK $\mathrm{TT}_{\mathrm{TT}}$ ) (termed session 1 in Fig. 1), the skiers used the classical technique (CL) for the first $3 \mathrm{~km}$, stopped for 2-min to change roller-skis and poles ("transition"), and then used the skating technique (SK) during the last $3 \mathrm{~km}$. The other time-trial $\left(\mathrm{SK}-\mathrm{SK} \mathrm{TT}_{\mathrm{TT}}\right)$ (termed session 2 in Fig. 1) was similar, except that the skating technique was employed for both $3-\mathrm{km}$ loops, with a 2-min stop in-between for a simulated change of roller-skis and poles.

The length and slope of the different segments of the 3-km loop were set preliminary to permit use of the 3 main gears of the classical [i.e., diagonal stride (DIA), kick-double pole (DPK), and double poling (DP)] and skating [i.e., gear 2 (G2), gear 3 (G3), and gear 4 (G4)] techniques (Fig. 2). The use of these specific gears was imposed on the basis of the slope. Skiers were not allowed to shift from one gear to another on the same segment of track or to shift from their strong to weak side (or vice versa) while utilizing the G2 or G4 techniques (Nilsson, Tveit, \& Eikrehagen, 2004). The course was configured to reproduce the demands imposed by a real XCS race as closely as possible. Two laser beams detected 


\begin{tabular}{|c|c|c|c|}
\hline $\begin{array}{c}\text { SESSION } 1 \\
\text { Preliminary Test } 1 \\
\left(\mathrm{CL}^{\left.-\mathrm{SK}_{\pi}\right)}\right.\end{array}$ & 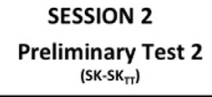 & 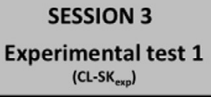 & $\begin{array}{c}\text { SESSION } 4 \\
\underset{\left(\text { SK-SK }_{\text {exp }}\right)}{\text { Experimental test } 2}\end{array}$ \\
\hline & & $\begin{array}{l}\text { MVC+EMG } \\
35 \% M V C+E M G\end{array}$ & $\begin{array}{l}\text { MVC+EMG } \\
35 \% M V C+E M G\end{array}$ \\
\hline $\begin{array}{c}\text { Warm-up } \\
1.5 \mathrm{~km} \mathrm{SK}+1.5 \mathrm{~km} \mathrm{CL}\end{array}$ & $\begin{array}{c}\text { Warm-up } \\
1.5 \mathrm{~km} \mathrm{CL}+1.5 \mathrm{~km} \mathrm{SK}\end{array}$ & $\begin{array}{c}\text { Warm-up } \\
1.5 \mathrm{~km} \mathrm{SK}+1.5 \mathrm{~km} \mathrm{CL} \\
\end{array}$ & $\begin{array}{c}\text { Warm-up } \\
1.5 \mathrm{~km} \mathrm{Cl}+1.5 \mathrm{~km} \mathrm{SK}\end{array}$ \\
\hline $\begin{array}{l}3 \mathrm{~km} \mathrm{CL} \\
\text { Resp. }\end{array}$ & $\begin{array}{l}3 \mathrm{~km} \text { SK } \searrow \\
\text { Resp. }\end{array}$ & $\begin{array}{l}3 \mathrm{~km} \mathrm{CL} \text { \& } \\
\text { Resp. }\end{array}$ & $\begin{array}{l}3 \mathrm{~km} \text { SK } \\
\text { Resp. }\end{array}$ \\
\hline Transition (2 min) & Transition (2 min) & $\begin{array}{l}\text { Transition ( } 2 \mathrm{~min}) \\
35 \% \mathrm{MVC}+\mathrm{EMG} \\
\text { BLa + RPE }\end{array}$ & $\begin{array}{l}\text { Transition ( } 2 \text { min) } \\
35 \% M V C+E M G \\
\text { BLa + RPE }\end{array}$ \\
\hline $\begin{array}{l}\mathbf{3} \mathbf{~ k m} \text { SK } \\
\text { Resp. }\end{array}$ & $\underset{\text { Resp. }}{\mathbf{3} \mathbf{~ k m} \text { SK } \bigwedge}$ & $\begin{array}{l}\mathbf{3} \text { km SK } \\
\text { Resp.+EMG dyn.+force } \backslash\end{array}$ & \begin{tabular}{|l|l}
$\mathbf{3}$ km SK \\
Resp.+EMG dyn.+force
\end{tabular} \\
\hline & & $\begin{array}{l}\text { 35\%MVC+EMG } \\
\text { BLa + RPE }\end{array}$ & $\begin{array}{l}35 \% M V C+E M G \\
B L a+R P E\end{array}$ \\
\hline \multicolumn{2}{|c|}{ Random order } & \multicolumn{2}{|c|}{ Random order } \\
\hline
\end{tabular}

Fig. 1. A schematic representation of the experimental design. MVC: maximum voluntary contractions; $35 \% \mathrm{MVC}$ : isometric contraction at $35 \%$ of the MVC; SK: skating technique; CL: classical technique; BLa: blood level of lactate; RPE: ratings of perceived exertion; Resp.: respiratory measurements; EMG dyn.: EMG measurements during the exercise; force: measurements of pole and plantar forces.

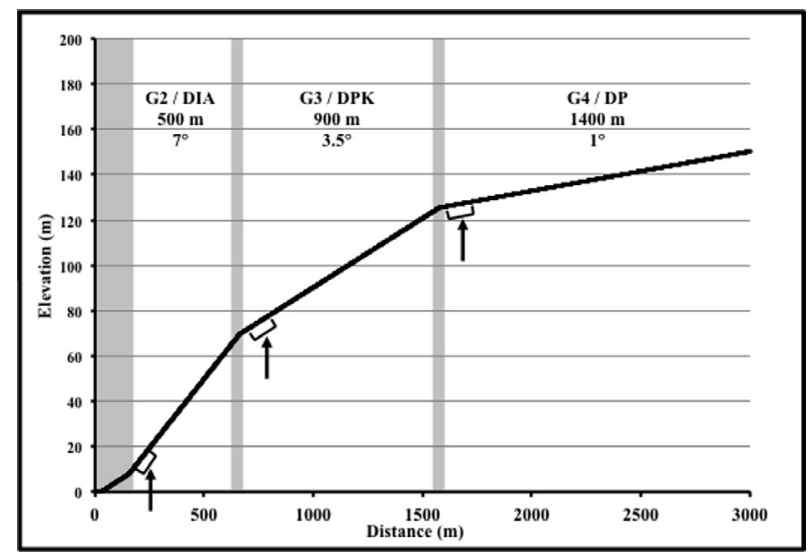

Fig. 2. Profile of the 3-km track. The gray areas represent the transition between two slopes, during which the skier must progressively adapt his technique to the new slope. These sections were not included in the data analysis. G2: skating gear 2; DIA: classical diagonal stride technique; G3: skating gear 3; KDP: classical kick double-poling technique; G4: skating gear 4; DP: classical double pole technique. The arrows represent the periods of measurement (EMG and pole and plantar forces) at the beginning of each segment of the second loop.

the position of the skier and adjusted the speed of the belt if the skier moved to the front (i.e., increasing speed) or rear (i.e., decreasing speed) of the belt.

\subsubsection{Experimental testing (sessions 3 and 4)}

Procedures similar to those for the first two TT sessions were employed during the third (CL-SK $\exp _{\text {) }}$ and fourth $\left(\mathrm{SK}_{-} \mathrm{SK}_{\exp }\right)$ sessions. Sessions 3 and 4 were also performed in random order, but at a 
pre-determined submaximal speed of $96 \%$ of the corresponding initial TT speed measured on each segment of the track (Fig. 2) for the first 3-km loop (i.e., 96\% of CL speed or 96\% of SK speed) and, for the second 3-km loop, 96\% of the mean SK speed during both sessions 1 and 2 . In this manner, the SK speed during the second 3-km loop was similar for sessions 3 and 4, allowing direct comparisons.

The blood level of lactate (BLa) and ratings of perceived exertion (RPE) were determined after each 3-km loop. In addition, maximal isometric voluntary contractions (MVCs) and EMG measurements on the vastus lateralis (VLa) and triceps brachii ( $\mathrm{TBr}$ ) muscles were performed prior to warm-up (T0). EMG monitoring at 35\% of MVC was also performed at T0, at the time of transition (Tr, immediately after taking off the skis and poles) and after the second 3-km loop (End). Respiratory data were collected continuously during both skiing loops. Dynamic EMG measurements on the VLa and TBr muscles, along with pole and plantar force data, were collected during the first 20-s of skiing on each segment of the second 3-km loop.

\subsection{Measurements}

The different periods of measurement are depicted in Figs. 1 and 2.

\subsubsection{Physiological and perceptual measurements}

During all sessions, respiratory variables were recorded continuously with the mixed expired procedure employing an ergo-spirometry system (AMIS 2001 model C, Innovision A/S, Odense, Denmark) equipped with an inspiratory flow meter. This system has been shown to be valid for testing elite athletes (Jensen, Jørgensen, \& Johansen, 2002). Expired $\mathrm{O}_{2}$ and $\mathrm{CO}_{2}$, breathing frequency (Bf) and the minute ventilation ( $\dot{\mathrm{VE}}$ ) were monitored continuously and averaged every $30 \mathrm{~s}$. Heart rate (HR) was monitored continuously using the HR monitor Polar S610 (Polar Electro Oy, Kempele, Finland) in combination with the metabolic cart.

During sessions 3 and 4, finger-tip capillary blood $(20 \mu \mathrm{L})$ was sampled immediately after the end of each 3-km loop for determination of BLa using a Biosen C-Line Sport Analyser (EKF Diagnostics, Magdeburg, Germany). At the same time, the RPE for overall exertion, the legs and the arms were assessed using the 6-20 Borg scale (Borg, 1970).

\subsubsection{Pole and plantar forces}

For both the classic and skating techniques during sessions 3 and 4, each skier used custom-made carbon-fiber racing poles adjusted to their preferred length. A 60-g strain gauge force transducer installed in a 75-g aluminum tube (Hottinger-Baldwin Messtechnik GmbH, Darmstadt, Germany) was mounted directly below the pole grip. This force transducer was positioned to measure ground reaction forces directed along the length of the pole.

Plantar ski reaction forces were recorded at $100 \mathrm{~Hz}$ using a Pedar mobile system (Novel GmbH, Munich, Germany). Validation and calibration of these systems for determination of the pole and plantar forces were achieved using procedures described elsewhere (Holmberg, Lindinger, Stöggl, Eitzlmair, \& Muller, 2005). The pole forces were sampled at $2000 \mathrm{~Hz}$, amplified (gain 1000; TeleMyo 2400T G2, Noraxon, Scottsdale, AZ, USA) and band-pass filtered (20-400 Hz; $20 \mathrm{~dB} /$ oct), transmitted telemetrically and simultaneously stored on a computer via an A/D converter card.

\subsubsection{EMG measurements}

During sessions 3 and 4, the EMG signals at the surface of the VLa and TBr muscles were recorded using pre-gelled bi-polar $\mathrm{Ag} / \mathrm{AgCl}$ surface electrodes (Skintact; Leonhard Lang $\mathrm{GmbH}$, Innsbruck, Austria).

Prior to electrode fixation, the skin was shaved, abraded lightly, degreased, and disinfected using alcohol swabs. For each muscle, two recording electrodes were positioned $30 \mathrm{~mm}$ apart parallel to the underlying fibers and over the belly, in accordance with international standards (Hermens, Freriks, Disselhorst-Klug, \& Rau, 2000). The reference electrode was attached to the lateral epicondyle of the humerus. EMG signals were recorded with TeleMyo 2400T G2 (input impedance $>10 \mathrm{MU}$, common mode rejection ratio $85 \mathrm{~dB}$, Noraxon, Scottsdale, AZ, USA) and the double-differential myoelectric 
signals amplified $(\times 2000)$ and bandpass-filtered $(10-500 \mathrm{~Hz})$ to remove noise (first-order high-pass $6 \mathrm{~dB} /$ oct slope; sixth-order Butterworth low-pass, $36 \mathrm{~dB} /$ oct slope). Data were sampled $(2000 \mathrm{~Hz})$ and stored on a computer.

The kinetic and EMG measuring equipment was carried in a hip belt during the third and fourth sessions of the experimental protocol. EMG and pole force data were collected by the same complete measuring system (TeleMyo 2400T G2, Noraxon, Scottsdale, AZ, USA). Synchronization of this data with the Pedar mobile system (plantar forces) was achieved by a signal produced when the latter started.

\subsubsection{Isometric muscle tests}

Before the warm-up for sessions 3 and 4, three 2-3-s maximal voluntary contractions (MVC) of the VLa and TBr muscles on the skier's weak side were performed to determine pre-trial maximal isometric force and peak EMG signal amplitudes. The skier sat on a modified chair during these trials and was allowed 2-min of recovery between contractions. In the case of the $\mathrm{TBr}$, the skier sat with the upper arm alongside the body and the elbow flexed at $90^{\circ}$, with the trunk and upper arms strapped to the back of the chair to minimize trunk movement during the contraction. The skier was asked to perform maximal elbow extension against a fixed resistance provided by a chain anchored to the ceiling and attached to his wrist. In the case of the VLa, each skier was asked to perform maximal knee extension while seated and stabilized with a waist belt. The knee was flexed at $90^{\circ}\left(180^{\circ}\right.$ representing a straight knee), with fixed resistance provided by a chair attached to the skier's ankle and anchored to the wall behind the chair.

Elbow and knee angles were measured at $2000 \mathrm{~Hz}$ by electrogoniometers (potentiometer: Megatron, Munich, Germany; strain gauges: Penny \& Giles Controls Ltd., Cmwfelinfach, UK) and collected with the same complete measuring system used to monitor EMG and pole force data (TeleMyo 2400T G2, Noraxon, Scottsdale, AZ, USA). The MVCs were recorded using a standard, calibrated strain gauge transducer (model 333A, K-TOYO, Seoul, Korea) inserted between the chain and the wrist or the ankle strap. Signals from this transducer were sampled at $100 \mathrm{kHz}$ and recorded linearly from 0 to $2500 \mathrm{~N}$ on a computerized system for measurement of muscle function (Muscle Lab 4010, Bosco System, Rieti, Italy).

After 10 min of passive recovery, the skier contracted each muscle (VLa and $\mathrm{TBr}$ ) isometrically for $4 \mathrm{~s}$ at $35 \%$ of his previously determined MVC (Hausswirth, Brisswalter, Vallier, Smith, \& Lepers, 2000). The force equivalent to $35 \%$ MVC was displayed in real-time on a computer monitor (using the dedicated software of the Muscle Lab 4010 system) placed in front of each skier. The EMG signals from these two sub-maximal isometric contractions were subsequently used as reference values for the non-fatigued state (T0).

\subsection{Data analysis}

\subsubsection{Aerobic energy expenditure (AEE)}

The aerobic energy expenditure (AEE, in $\mathrm{kJ} \mathrm{min}^{-1}$ ) was determined from the average of the mean values for $\mathrm{VO}_{2}$ and RER during the final minute of each track segment (Fig. 2) using the tables developed by Lusk (1928) (McLean \& Tobin, 1987; Sidossis, Horowitz, \& Coyle, 1992).

\subsubsection{Kinematic and kinetic analysis}

For sessions 1 and 2, the mean speed for each segment of the second 3-km loop was recorded for each skier.

The mean values of peak plantar and pole forces, poling time (between initial pole contact and pole take-off), cycle length (poling time $\times$ velocity) and cycle rates for 10-15 successive cycles during the first $20 \mathrm{~s}$ of each segment of the second 3-km loop were processed and used for further analysis. The poling phase of the upper limb was identified from the poling force profile. The start and end of the poling phase were defined as a rise in force above $15 \%$ and a fall below $10 \%$, respectively, of the mean force during the 20-s period of measurement (Wakeling, Blake, \& Chan, 2010). Employing the plantar forces instead, the same approach was applied to identify the start and end of the lower-limb 
propulsive phase (i.e., the eccentric and concentric phases) during use of the $\mathrm{G} 2$ technique. In contrast, with G3 and G4, the lower-limb propulsive phase was identified by combining information about the plantar force and displacement of the knee angle, in order to avoid interference from a sliding phase (when the lower limb is supporting body weight) which occurs prior to propulsion. The beginning of the propulsive phase of the lower limb occurred when the knee angle reached a maximum for the first time after foot contact [i.e., the beginning of the eccentric phase (Millet, Perrey, Candau, \& Rouillon, 2002)], while this phase ended when foot contact was lost.

\subsubsection{EMG analysis of isometric MVC and submaximal isometric contractions}

The raw EMG signals were band-pass filtered digitally $(10-400 \mathrm{~Hz}$, Butterworth second-order) to remove both low- and high-frequency noise that was not completely suppressed by the analogue band-pass filter. The cut-off frequency was chosen by visual inspection of the spectrum of the signal power. The density function of this power spectrum was derived using fast Fourier transformation.

The EMG signals were then full-wave rectified and a root mean square (RMS) value calculated from consecutive 0.5-s time windows. The RMS values and mean power frequency (MPF) (obtained from the power spectrum using standard equations (Kwatnyn, Thomas, \& Kwatny, 1970), were the primary variables. These values for the submaximal isometric contractions were expressed as percentages of the corresponding values for the non-fatigued state [i.e., the 35\% MVC test performed at T0 (Hausswirth et al., 1996)].

For the dynamic contractions, at least 10 consecutive cycles of movement recorded during the 20-s biomechanical measurements on each segment of track were analysed. The EMG signals from the $\mathrm{TBr}$ and VLa muscles during the poling and kicking phases (as defined previously) were band-pass filtered, full-wave rectified and analysed. The RMS values for both the TBr and VLa muscles were calculated following the same procedure as for the isometric contractions. For both muscles, the average RMS values for the 10 consecutive cycles on each segment of track were normalized to the highest RMS value during the three MVC trials, and expressed as a percentage of the MVC (\%MVC).

\subsection{Statistical analyses}

All results are presented as means \pm standard deviations (SD). The speeds recorded during the second phase of the time-trial sessions were analysed using a two-way [condition (CL-SK $\mathrm{TT}_{\mathrm{TT}}$ or SK-SK $\left.\mathrm{TT}_{\mathrm{TT}}\right) \times$ gear (G2, G3 and G4)] repeated-measures analysis of variance (ANOVA). The dynamic EMG RMS values were analysed using a two-way [condition (CL-SK $\mathrm{exp}_{\text {or } \mathrm{SK}-\mathrm{SK}}$ exp $) \times \operatorname{gear}(\mathrm{G} 2$, G3 and G4)] ANOVA. All parameters studied in relationship to the various time-points (TO, Tr, and End) and conditions during the testing sessions (CL-SKexp or SK-SKexp) were analysed by means of a two-way [condition $\left(\mathrm{CL}-\mathrm{SK}_{\exp }\right.$ or $\left.\mathrm{SK}-\mathrm{SK}_{\exp }\right) \times$ time $(\mathrm{T} 0, \mathrm{Tr}$ and End)] ANOVA. If the data were not normally distributed and/or demonstrated unequal variance (as revealed by the Kolmogorov-Smirnov test) an ANOVA on ranks was systematically performed. When an overall effect was observed, the Holm-Sidak post hoc test was used to make pair-wise comparisons. A $p$-value of $<.05$, as determined with version 3.5 of the Sigma Stat software package (St Louis, MO, USA), was considered statistically significant.

\section{Results}

\subsection{Speed during the preliminary testing sessions}

During the second 3-km loop of the preliminary testing sessions, the G4 skating speed was significantly higher during SK-SK $\mathrm{TT}_{\mathrm{TT}}$ than during $\mathrm{CL}_{-} \mathrm{SK}_{\mathrm{TT}}\left(26.2 \pm 1.7\right.$ versus $25.2 \pm 1.9 \mathrm{~km} \mathrm{~h}^{-1}$, respectively; $p=.016)$. Although not statistically significant, the same trend was observed with the G3 skating technique (15.1 \pm 1.5 versus $14.4 \pm 1.2 \mathrm{~km} \mathrm{~h}^{-1}$, respectively; $p=.056$ ). No such difference was observed with the $\mathrm{G} 2$ technique (10.7 \pm 1.4 versus $10.6 \pm 1.1 \mathrm{~km} \mathrm{~h}^{-1}$, respectively; $p=.82$ ). 
Table 1

Physiological responses and ratings of perceived exertion in six elite male cross-country skiers during sessions 3 and 4 .

\begin{tabular}{|c|c|c|c|c|c|c|c|c|c|c|c|}
\hline & & $\dot{\mathrm{VO}}_{2}\left(\mathrm{~L} \mathrm{~min}^{-1}\right)$ & AEE $\left(\mathrm{kJ} \mathrm{min}{ }^{-1}\right)$ & HR (bpm) & $\dot{\mathrm{VE}}\left(\mathrm{L} \mathrm{min}^{-1}\right)$ & Bf (breaths $\min ^{-1}$ ) & RER & $\mathrm{BLa}\left(\mathrm{mmol} \mathrm{L}^{-1}\right)$ & RPE breath & RPE arms & RPE legs \\
\hline \multirow[t]{2}{*}{ CL-SK $\exp$} & First 3 km loop & $4.4 \pm 0.4$ & $93.6 \pm 10.3$ & $168.5 \pm 9.6^{\#}$ & $138.3 \pm 25.1$ & $41.5 \pm 7.9^{* \#}$ & $1.02 \pm 0.1^{\#}$ & $8.3 \pm 2.6$ & $14.2 \pm 1.0^{* \#}$ & $13.8 \pm 1.7$ & $14.5 \pm 2.0^{\# \# \#}$ \\
\hline & Second $3 \mathrm{~km}$ loop & $4.3 \pm 0.3^{*}$ & $90.2 \pm 6.8^{*}$ & $176.2 \pm 6.7$ & $142.5 \pm 22.4$ & $46.2 \pm 9.2$ & $0.98 \pm 0.0$ & $7.2 \pm 3.2$ & $15.8 \pm 0.8$ & $15.0 \pm 1.3$ & $16.2 \pm 1.7$ \\
\hline \multirow[t]{2}{*}{ SK-SK $\exp$} & First 3 km loop & $4.4 \pm 0.3$ & $94.5 \pm 7.0$ & $169.4 \pm 3.5^{\#}$ & $146.9 \pm 22.4$ & $46.7 \pm 9.9$ & $1.02 \pm 0.0^{\#}$ & $6.9 \pm 1.6^{\#}$ & $15.3 \pm 1.0$ & $14.5 \pm 2.0$ & $15.0 \pm 1.4$ \\
\hline & Second $3 \mathrm{~km}$ loop & $4.2 \pm 0.3$ & $88.9 \pm 6.2$ & $175.8 \pm 4.3$ & $138.9 \pm 22.3$ & $45.0 \pm 9.1$ & $0.98 \pm 0.0$ & $6.4 \pm 1.2$ & $15.5 \pm 1.5$ & $15.0 \pm 1.5$ & $16.0 \pm 1.1$ \\
\hline
\end{tabular}

$\mathrm{VO}_{2}$ : oxygen uptake; AEE: aerobic energy expenditure; HR: heart rate; $\dot{V E}$ : minute ventilation; RER: respiratory exchange ratio; BLa: blood lactate concentration; RPE: rate of perceived exertion.

\#,\#\#Significant differences between the first and the second 3-km loop at $p<.05$ and $p<.001$, respectively.

* Significant differences between CL-SK $\mathrm{exp}_{\text {and }}$ SK-SK ${ }_{\exp }$ conditions at $p<.05$. 


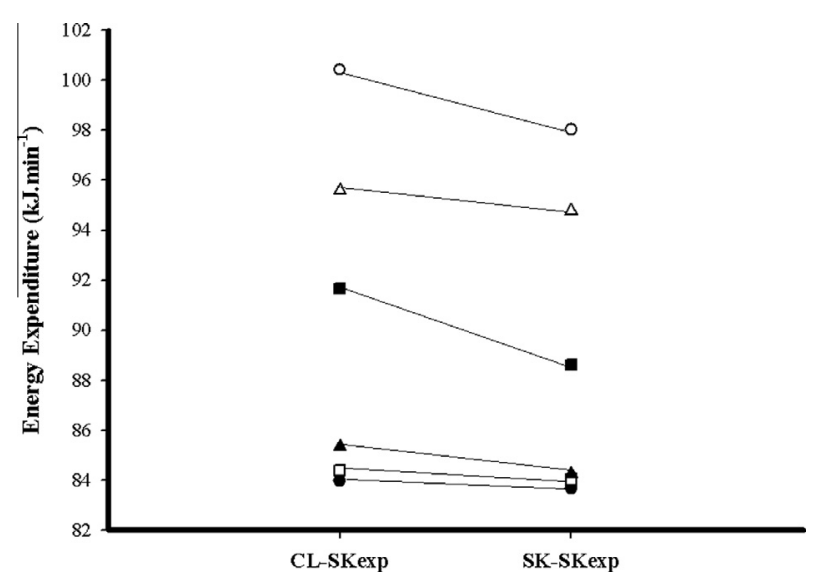

Fig. 3. Individual changes in energy expenditure during the second $3-\mathrm{km}$ loop of the $\mathrm{CL}-\mathrm{SK}_{\exp }$ and $\mathrm{SK}-\mathrm{SK}_{\exp }$ trials. Each symbol represents one individual skier. Note that all skiers exhibited higher energy expenditure during CL-SK $\mathrm{exp}_{\text {. }}$

\subsection{Physiological findings (Table 1)}

$\dot{\mathrm{VO}}_{2}$ and the aerobic energy expenditure (AEE) during the second 3-km loop of experimental sessions 3 and 4 were significantly higher $\left(p=.037\right.$ and $p=.039$, respectively) during CL-SK exp $_{\text {than }}$ SK-SK exp (Fig. 3). During CL-SKexp, the frequency of breathing (Bf) was higher at the end of the experimental session (End) than during the transition $(\operatorname{Tr})(p=.028)$, during which phase Bf was higher for SK-SKexp than CL-SK $\exp (p=.034)$. In the case of both CL-SK ${ }_{\exp }$ and SK-SK exp, $\dot{V O}_{2}$ and AEE $(p=.001$ in both cases) and RER ( $p=.007$ for CL-SK $\mathrm{exp}_{\text {and }}$ an $=.004$ for $\left.\mathrm{SK}_{-} \mathrm{SK}_{\exp }\right)$ were greater during the first than the second 3-km loop. On the other hand, HR was higher during the second loop ( $p=.037$ for CL-SK $\mathrm{exp}$ and $p=.013$ for $\mathrm{SK}_{-} \mathrm{SK}_{\mathrm{exp}}$ ). Finally, during $\mathrm{SK}-\mathrm{SK}_{\mathrm{exp}}$, BLa was greater at Tr than End.

\subsection{RPE (Table 1)}

At Tr, the RPE for breathing was greater during SK-SK $\exp _{\text {p }}$ than $\mathrm{CL}-\mathrm{SK}_{\exp }(p=.017)$, whereas no dif-

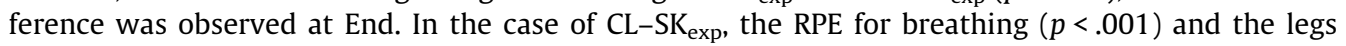
$(p=.027)$ were greater at End than $\operatorname{Tr}(p<.001)$ with no such differences during SK-SK exp.

\section{4. $E M G$}

\subsubsection{Isometric submaximal contractions}

The RMS for VLa was significantly greater at both $\operatorname{Tr}(p=.004)$ and End $(p=.012)$ during CL-SK $\exp$

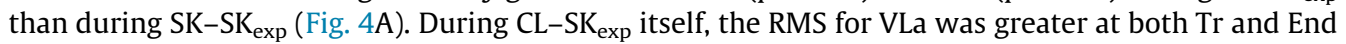

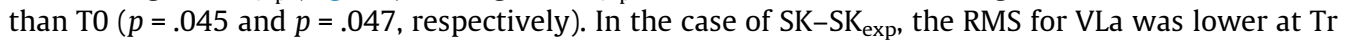
than T0 $(p=.025)$. No such differences were observed for the TBr muscle (Fig. 4B) and there was no difference between the MPF values for the VLa (Fig. 4C) and TBr (Fig. 4D) muscles.

\subsubsection{Dynamic contractions}

The RMS of the VLa muscle while G3 and G4 skating was significantly greater during CL-SK $\mathrm{exp}_{\text {than }}$ SK-SK $K_{\exp }(p<.001)$ (Fig. 5A). During SK-SK exp itself, the RMS for VLa was greater with the G2 than G3. In the case of CL-SK exp, the RMS for VLa was greater when using the G4 than the G3 technique. No such differences were observed for the $\mathrm{TBr}$ muscle (Fig. 5B). 

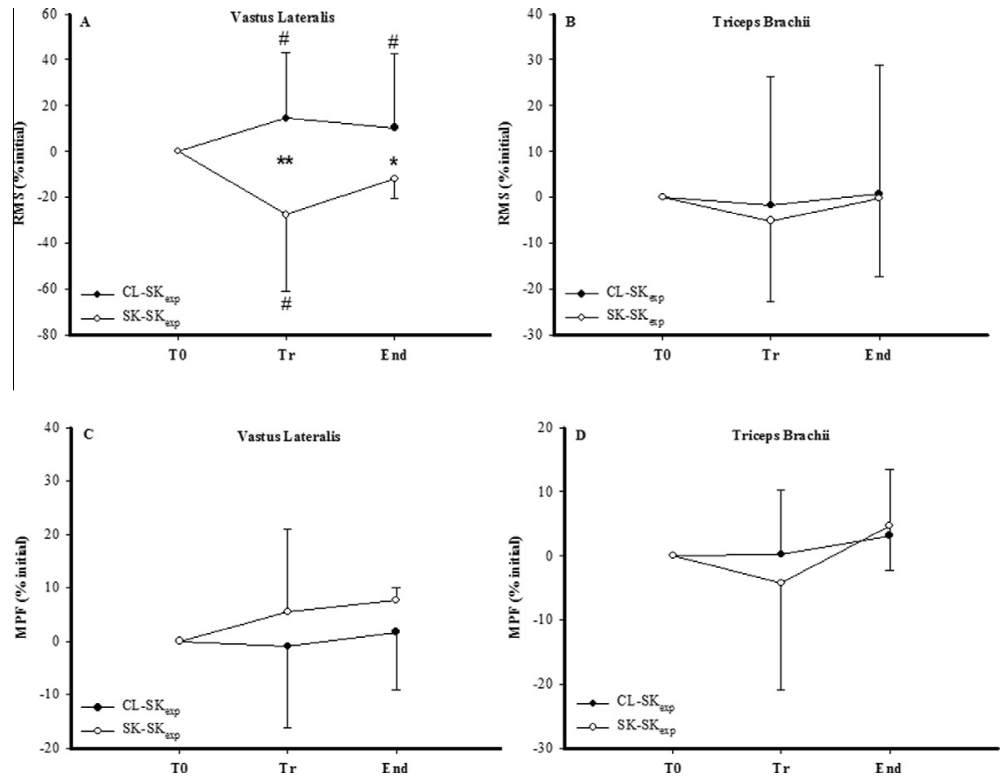

Fig. 4. Alterations in the signal amplitude (RMS) and mean power frequency (MPF) of the vastus lateralis (A and C, respectively) and triceps brachii (B and D, respectively) muscles. T0: during the isometric contractions prior to exercise. Tr: during the transition. End: at the end of the exercise. The filled circles depict the CL-SK $\exp$ trials and the open circles SK-SK $\mathrm{exp}^{* * * *} p<.05$ and $p<.01$, respectively, for $\mathrm{CL}-\mathrm{SK}_{\exp }$ in comparison to $\mathrm{SK}_{-\mathrm{SK}}{ }_{\text {exp }}{ }^{*} p<.05$ in comparison to T0.
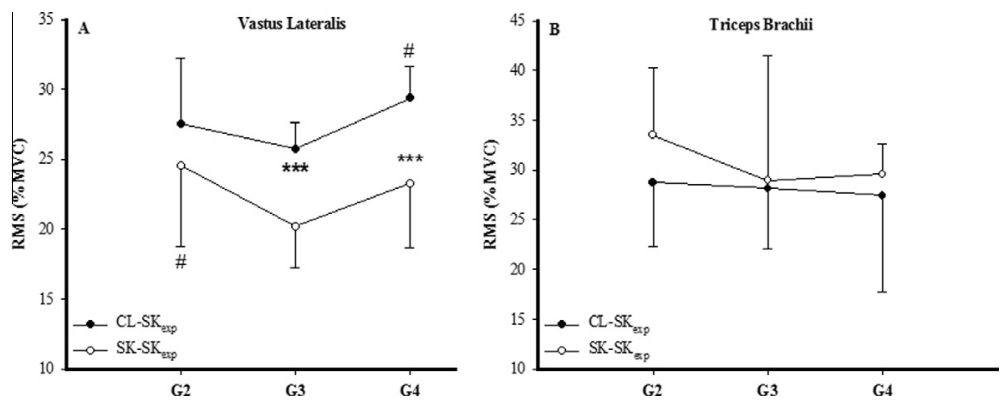

Fig. 5. Alterations in the signal amplitude (RMS) of the vastus lateralis (A) and triceps brachii (B) muscles during the poling phase of cycles performed during the first $20 \mathrm{~s}$ of G2, G3 and G4 skating in the second 3-km loop of the experimental sessions 3 and 4. The filled circles depict the CL-SK $\exp$ trial and the open circles SK-SK exp $^{* * *} p<.001$ for CL-SKexp in comparison to SK-SKexp. $\#, \# \# p<.05$ and $p<.01$, respectively, in comparison to G3.

\subsection{Kinetic and kinematic findings (Table 2)}

Peak plantar and pole forces, poling times, cycle length and cycle rates did not differ significantly

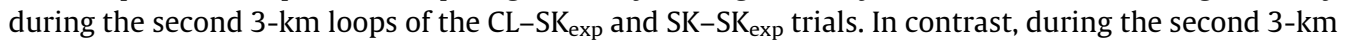
loops of the experimental sessions 3 and 4, there were significant main effect differences between the skating sub-techniques with respect to cycle rates $(\mathrm{G} 3>\mathrm{G} 2>\mathrm{G} 4 ; p<.001)$, cycle lengths (G4 > G3 > G2; $p<.001)$. During the G2 segment, peak poling forces were significantly lower than during the G3 and G4 segments ( $p<.001)$ and poling time was significantly longer than during the G3 and G4 segments $(p<.001)$. 
Table 2

Kinetic and kinematic variables during the second 3-km loop of sessions 3 and 4 .

\begin{tabular}{|c|c|c|c|c|c|c|c|c|c|c|c|c|c|c|c|}
\hline & \multicolumn{3}{|c|}{ Peak poling force $(\mathrm{N})$} & \multicolumn{3}{|c|}{ Peak plantar force $(\mathrm{N})$} & \multicolumn{3}{|c|}{ Cycle length $(\mathrm{m})$} & \multicolumn{3}{|c|}{ Cycle rate $(\mathrm{Hz})$} & \multicolumn{3}{|c|}{ Poling time $(\mathrm{s})$} \\
\hline & G2 & G3 & G4 & G2 & G3 & G4 & G2 & G3 & G4 & G2 & G3 & G4 & G2 & G3 & G4 \\
\hline CL-SK $K_{\exp }$ & $\begin{array}{l}134.3 \pm \\
31.6^{\# \#, \$ \$}\end{array}$ & $\begin{array}{l}182.2 \pm \\
58.7^{\$ \$}\end{array}$ & $\begin{array}{l}173.4 \pm \\
83.5\end{array}$ & $\begin{array}{l}954.1 \pm \\
189.7\end{array}$ & $\begin{array}{l}918.7 \pm \\
215.0\end{array}$ & $\begin{array}{l}944.7 \pm \\
321.4\end{array}$ & $\begin{array}{l}3.63 \pm \\
0.26^{\# \#, \$ \$}\end{array}$ & $\begin{array}{l}3.90 \pm \\
0.55^{\$ \$}\end{array}$ & $\begin{array}{l}11.38 \pm \\
0.93\end{array}$ & $\begin{array}{l}0.72 \pm \\
0.02^{\# \#, \$ \$}\end{array}$ & $\begin{array}{l}0.94 \pm \\
0.04^{\$ \$}\end{array}$ & $\begin{array}{l}0.59 \pm \\
0.04\end{array}$ & $\begin{array}{l}0.57 \pm \\
0.07^{\# \#, \$ \$}\end{array}$ & $\begin{array}{l}0.36 \pm \\
0.04\end{array}$ & $\begin{array}{l}0.30 \pm \\
0.05\end{array}$ \\
\hline SK-SK $\mathrm{exp}$ & $\begin{array}{l}131.3 \pm \\
19.9^{\# \#, \$ \$}\end{array}$ & $\begin{array}{l}177.2 \pm \\
18.3^{\$ \$}\end{array}$ & $\begin{array}{l}170.8 \pm \\
37.0\end{array}$ & $\begin{array}{l}859.9 \pm \\
143.1\end{array}$ & $\begin{array}{l}823.7 \pm \\
87.9\end{array}$ & $\begin{array}{l}874.9 \pm \\
137.4\end{array}$ & $\begin{array}{l}3.64 \pm \\
0.28^{\# \#, \$ \$}\end{array}$ & $\begin{array}{c}3.95 \pm \\
0.52^{\$ \$}\end{array}$ & $\begin{array}{l}11.72 \pm \\
0.70\end{array}$ & $\begin{array}{l}0.72 \pm \\
0.03^{\# \#, \$ \$}\end{array}$ & $\begin{array}{l}0.93 \pm \\
0.05^{\$ \$}\end{array}$ & $\begin{array}{l}0.58 \pm \\
0.04\end{array}$ & $\begin{array}{l}0.57 \pm \\
0.07^{\# \#, \$ \$}\end{array}$ & $\begin{array}{l}0.37 \pm \\
0.02\end{array}$ & $\begin{array}{l}0.35 \pm \\
0.16\end{array}$ \\
\hline
\end{tabular}

G2, G3 and G4: the three main gears of the skating technique: gear 2, gear 3 and gear 4 .

\#\# Significantly different from G3, $p<.001$.

$\$ \$$ Significantly different from G4, $p<.001$. 


\section{Discussion}

As we hypothesized, AEE during the second 3-km loop of the CL-SK ("skiathlon") was higher $(+1.5 \%)$ than when the same skating exercise was performed with no change in skiing technique (SK-SK). This difference cannot be explained by fatigue, since the physiological demands during the first 3-km loops of sessions 3 and 4 were identical. In addition, skating speed during CL-SK $\mathrm{TT}_{\mathrm{TT}}$ was slower than during $\mathrm{SK}_{-} \mathrm{SK}_{\mathrm{TT}}$, confirming that the prior performance of classical skiing affected subsequent skating performance.

These results are analogous to certain previous findings that during a triathlon the energy cost of running is $1.6-11.6 \%$ higher following a transition from cycling to running than with running alone (Guezennec et al., 1996; Hausswirth et al., 1996; Hue et al., 1998; Kreider et al., 1988). This increase has been related to alterations in biomechanics, e.g., an enhanced variability in step frequency (Candau et al., 1998). However, in the present investigation, the peak pole and plantar forces, along with cycle rates did not differ during the second 3-km loop of skating, regardless of whether the skier had employed the skating ( $\mathrm{SK}-\mathrm{SK}_{\exp }$ ) or classical $\left(\mathrm{CL}-\mathrm{SK}_{\exp }\right)$ technique during the first loop. These findings on biomechanical parameters thus disprove this aspect of our hypothesis.

During the transition triathletes have been observed to lose coordination during running (Quigley \& Richards, 1996), a phenomenon attributed to the change from the typical frequency of cycling to that of running (1.5-2.0 to 1-1.5 Hz). Moreover, Snyder and colleagues (2012) showed that running in humans who must adjust their step frequency (cycle rate) from an imposed to a freely chosen rate, pre-programed optimization processes are designed to minimize the energy cost. In our experiment, the skiers made the transition between skiing techniques employing distinctly different patterns of locomotion (and probably cycle rates as well) and thus, one may hypothesize that the energy cost would be elevated until they regain their metabolically optimal gait. However, Bilodeau and colleagues (1992) have shown that the cycle rates associated with different skating and classical techniques during uphill and flat skiing were universally quite similar. Thus, this mechanism cannot explain the alteration in AEE observed in the present case.

Perturbation of the EMG activity of the leg muscles is another explanation proposed for the increase in the energy cost of running after the cycling-to-running transition in triathlon events, even with unaltered leg kinetics (Chapman et al., 2009). This perturbation has been related to the change from concentric muscular contraction during cycling to the stretch-shortening cycle of activity when running, as well as to an alteration in the recruitment of motor units (Witt, 1993). Here, we also document perturbations in EMG activity, with an increase in the RMS of the VLa muscle during both iso-

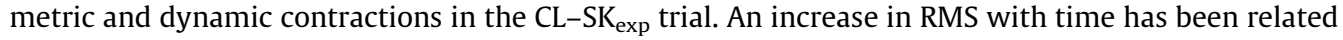
to muscle fatigue after prolonged running and after a simulated triathlon (Hausswirth et al., 2000). The authors of the latter investigation suggested that runners do not obviously modify their running mechanics in response to fatigue, but instead that the recruitment of motor units could be modified at the very beginning of a fatigued state.

However, in the present study, the alteration in EMG activity cannot be explained by a switch from concentric to plyometric contraction, since stretch-shortening of the leg extensor muscles occurs with both the classical (Komi \& Norman, 1987) and skating (Perrey, Millet, Candau, \& Rouillon, 1998) techniques of XCS. Rather, an important distinction between the skating and classical techniques of diagonal stride and kick-double poling is the manner in which propulsive forces are applied through the skis. The classical techniques involve a kick directed downward and posteriorly to drive the skier forward and during this kick, the ski must momentarily come to a complete stop. As the skiing velocity increases, the kick-off time is reduced so that the speed of muscle contraction needs to be higher. In contrast, with the skating techniques, ski forces are applied over a significantly longer period, since the ski is still gliding forward, and the speed of muscle contraction does not appear to be a limiting factor (Bilodeau et al., 1992).

In addition, the muscular demands made by the classical and skating techniques differ somewhat. Since the rate of muscle contraction is thought to influence muscular efficiency (Wakeling et al., 2010), the differences between the classical and skating techniques with respect to the application of ski forces can probably be explained by the slightly higher skating AEE immediately after a bout of skiing 
with the classical technique. In contrast to our findings on the leg extensor muscles, no difference in arm muscle fatigue between the second loops of the two trials was observed (i.e., the EMG of the TBr muscle did not change during the classic portion of the "skiathlon"). This could be explained by the negligible difference in the manner in which propulsive forces are applied through the poles in CL and SK, in contrast to how these forces act on the legs, as described above.

An alternative explanation based on observations of limb coordination suggests that while performing two novel tasks sequentially, our central nervous system employs a pattern of movement that allows both tasks, without being specific for either (Karniel \& Mussa-Ivaldi, 2002). This previous study revealed that the after-effects of the first task exert an impact on performance during the subsequent task. The timing or sequencing used in the present investigation is analogous to the manner in which skiers must adjust to the SK technique immediately after CL skiing. Whether the skiathlon involves the same cross-over effect of movement patterns (via muscle activation) observed from cycling to running in triathlon studies (Chapman, Vicenzino, Blanch, Dowlan, \& Hodges, 2008) as indicated by the alterations in muscle activation documented here, remains to be explored further, as does the reason why only the legs are affected.

It is important to emphasize that an actual skiathlon competition for men covers a total distance of $30 \mathrm{~km}$. Accordingly, our experimental conditions involved a much shorter distance, as well as a longer recovery period during the transition (which is only $\sim 30$ s during real XCS races). We can easily imagine that the impact of the classical skiing on AEE, neuromuscular function and discomfort (as commonly reported by skiers after the transition) would be greater with longer exercise and a shorter transition.

Moreover, although roller-skiing is intended to simulate XCS on snow as closely as possible, the classical technique is easier to perform using roller-skis than on snow. Indeed, one of the wheels of the roller-skis has a seamless clutch that only allows forward rotation, making it possible for the skier to kick and glide when performing the classical technique in much the same manner as a skier would use grip wax to kick off snow. However, this clutch never fails and requires less energy and muscular strain than skiing on snow. This absolute grip prevents the roller skis from slipping backwards, unlike the case of snow-skiing, where focusing on the appropriate technique to achieve sufficient grip is essential (Ainegren, Carlsson, Laaksonen, \& Tinnsten, 2014).

An additional limitation to the present investigation is that the slight differences observed in AEE might have been due to variability in the measurement of $\dot{\mathrm{VO}}_{2}$. However, even though the sample size was small, this difference was observed for every individual skier (Fig. 2) in this homogeneous group and the random order of measurement was designed to circumvent such possible bias. In addition, assessment of the technical error measurement [\%TEM] of our metabolic cart with the paired $t$-test indicated no differences in $\mathrm{VO}_{2}$ between the two days, with a \%TEM of $1.1 \%$, in comparison to the $1.5 \%$ mean difference in AEE.

\section{Conclusions}

This study documents for the first time alterations in skating AEE, performance (i.e., speed during time-trials) and neuromuscular function induced by the preceding classical portion of a skiathlon event. In connection with such competitions, the ability to link the classical and skating sections efficiently (i.e., with less pronounced alterations after transition) is of considerable practical interest, since the final outcome is often decided in the last sprint, during which the slightest detail can be decisive. Indeed, various authors have proposed that the extent to which AEE is perturbed during triathlon running reflects the level of the triathlete's ability (Hue et al., 1998; Millet \& Vleck, 2000). However, additional research is clearly needed in order to incorporate specific transition training into the programs of elite skiers in an optimal manner. Moreover, although we attempted to simulate the constraints imposed by an actual XCS race with the course on the treadmill, further research with longer duration of exercise and on snow is needed to confirm our present findings. If these alterations are confirmed, it would be of interest to examine whether the skier is able to recover while skating or whether this altered state persists until the end of the race. 


\title{
Acknowledgements
}

\author{
This study was supported financially by the Swedish National Centre for Research in Sports and the \\ Swedish Olympic Committee. The authors would like to thank the participants and their coaches sin- \\ cerely for their valuable cooperation and participation in this study.
}

\section{References}

Ainegren, M., Carlsson, P., Laaksonen, M. S., \& Tinnsten, M. (2014). The influence of grip on oxygen consumption and leg forces when using classical style roller skis. Scandinavian Journal of Medicine E Science in Sports, 24, 301-310.

Bilodeau, B., Boulay, M. R., \& Roy, B. (1992). Propulsive and gliding phases in four cross-country skiing techniques. Medicine and Science in Sports and Exercise, 24, 917-925.

Borg, G. (1970). Perceived exertion as an indicator of somatic stress. Scandinavian Journal of Rehabilitation Medicine, 2 , 92-98.

Candau, R., Belli, A., Millet, G. Y., Georges, D., Barbier, B., \& Rouillon, J. D. (1998). Energy cost and running mechanics during a treadmill run to voluntary exhaustion in humans. European Journal of Applied Physiolology and Occupational Physiology, 77, 479-485.

Cavanagh, P. R., \& Williams, K. R. (1982). The effect of stride length variation on oxygen uptake during distance running. Medicine and Science in Sports and Exercise, 14, 30-35.

Chapman, A. R., Vicenzino, B., Blanch, P., Dowlan, S., \& Hodges, P. W. (2008). Does cycling effect motor coordination of the leg during running in elite triathletes? Journal of Science and Medicine in Sport, 11, 371-380.

Chapman, A. R., Vicenzino, B., Hodges, P. W., Blanch, P., Hahn, A. G., \& Milner, T. E. (2009). A protocol for measuring the direct effect of cycling on neuromuscular control of running in triathletes. Journal of Sports Sciences, 27, 767-782.

Guezennec, C. Y., Vallier, J. M., Bigard, A. X., \& Durey, A. (1996). Increase in energy cost of running at the end of a triathlon. European Journal of Applied Physiolology and Occupational Physiology, 73, 440-445.

Hausswirth, C., Bigard, A. X., Berthelot, M., Thomaidis, M., \& Guezennec, C. Y. (1996). Variability in energy cost of running at the end of a triathlon and a marathon. International Journal of Sports Medicine, 17, 572-579.

Hausswirth, C., Bigard, A. X., \& Guezennec, C. Y. (1997). Relationships between running mechanics and energy cost of running at the end of a triathlon and a marathon. International Journal of Sports Medicine, 18, 330-339.

Hausswirth, C., Brisswalter, J., Vallier, J. M., Smith, D., \& Lepers, R. (2000). Evolution of electromyographic signal, running economy, and perceived exertion during different prolonged exercises. International Journal of Sports Medicine, 21, 429-436.

Heiden, T., \& Burnett, A. (2003). The effect of cycling on muscle activation in the running leg of an Olympic distance triathlon. Sports Biomechanics, 2, 35-49.

Hermens, H. J., Freriks, B., Disselhorst-Klug, C., \& Rau, G. (2000). Development of recommendations for SEMG sensors and sensor placement procedures. Journal of Electromyography and Kinesiology, 10, 361-374.

Holmberg, H. C., Lindinger, S., Stöggl, T., Eitzlmair, E., \& Muller, E. (2005). Biomechanical analysis of double poling in elite crosscountry skiers. Medicine and Science in Sports and Exercise, 37, 807-818.

Hue, O., Le Gallais, D., Chollet, D., Boussana, A., \& Prefaut, C. (1998). The influence of prior cycling on biomechanical and cardiorespiratory response profiles during running in triathletes. European Journal of Applied Physiolology and Occupational Physiology, 77, 98-105.

Jensen, K., Jørgensen, S., \& Johansen, L. (2002). A metabolic cart for measurement of oxygen uptake during human exercise using inspiratory flow rate. European Journal of Applied Physiolology, 87, 202-206.

Karniel, A., \& Mussa-Ivaldi, F. A. (2002). Does the motor control system use multiple models and context switching to cope with a variable environment? Experimental Brain Research, 143, 520-524.

Komi, P. V., \& Norman, R. W. (1987). Preloading of the thrust phase in cross-country skiing. International Journal of Sports Medicine, 8(Suppl 1), 48-54.

Kreider, R. B., Boone, T., Thompson, W. R., Burkes, S., \& Cortes, C. W. (1988). Cardiovascular and thermal responses of triathlon performance. Medicine and Science in Sports and Exercise, 20, 385-390.

Kwatnyn, E., Thomas, D. H., \& Kwatny, H. G. (1970). An application of signal processing techniques to the study of myoelectric signals. IEEE Transactions on Bio-medical Engineering, 17, 303-312.

Lusk, G. (1928). The elements of the science of nutrition. Philadelphia: Saunders.

McLean, J. A., \& Tobin, G. (1987). Animal and human calorimetry. New York: Cambridge.

Millet, G. P., Millet, G. Y., Hofmann, M. D., \& Candau, R. B. (2000). Alterations in running economy and mechanics after maximal cycling in triathletes: Influence of performance level. International Journal of Sports Medicine, 21, 127-132.

Millet, G. Y., Perrey, S., Candau, R., \& Rouillon, J. D. (2002). Relationships between aerobic energy cost, performance and kinematic parameters in roller ski skating. International Journal of Sports Medicine, 23, 191-195.

Millet, G. P., \& Vleck, V. E. (2000). Physiological and biomechanical adaptations to the cycle to run transition in Olympic triathlon: Review and practical recommendations for training. British Journal of Sports Medicine, 34, 384-390.

Nilsson, J., Tveit, P., \& Eikrehagen, O. (2004). Effects of speed on temporal patterns in classical style and freestyle cross-country skiing. Sports Biomechanics, 3, 85-107.

Perrey, S., Millet, G. Y., Candau, R., \& Rouillon, J. D. (1998). Stretch-shortening cycle in roller ski skating: Effects of technique. International Journal of Sports Medicine, 19, 513-520.

Quigley, E. J., \& Richards, J. G. (1996). The effects of cycling on running mechanics. Journal of Applied Biomechanics, 12, 470-479.

Sidossis, L. S., Horowitz, J. F., \& Coyle, E. F. (1992). Load and velocity of contraction influence gross and delta mechanical efficiency. International Journal of Sports Medicine, 13, 407-411. 
Snyder, K. L., Snaterse, M., \& Donelan, J. M. (2012). Running perturbations reveal general strategies for step frequency selection. Journal of Applied Physiology, 12, 1239-1247.

Wakeling, J. M., Blake, O. M., \& Chan, H. K. (2010). Muscle coordination is key to the power output and mechanical efficiency of limb movements. The Journal of Experimental Biology, 213, 487-492.

Witt, M. (1993). Co-ordination of leg muscles during cycling and running in triathlon. XIVth Congress of International Society of Biomechanics, 1470-14711. 